Artículo

\title{
Fertilización orgánica con tres niveles de gallinaza en cuatro cultivares de papa
}

\author{
Josué Peñaloza Monroy ${ }^{1}$ \\ Ana Karen Reyes Ramírez ${ }^{1}$ \\ Andrés González Huerta ${ }^{1}$ \\ Delfina de Jesús Pérez López ${ }^{1 \S}$ \\ Dora Ma. Sangerman-Jarquín ${ }^{2}$
}

${ }^{1}$ Facultad de Ciencias Agrícolas-Universidad Autónoma del Estado de México-Campus Universitario 'El Cerrillo'. El Cerrillo Piedras Blancas, Toluca, México. CP. 50200. Tel. 722 2965518, ext. 148 (agonzalezh@uaemex.mx; josuepm_30@hotmail.com; anakaren_reyes26@hotmail.com). ${ }^{2}$ Campo Experimental Valle de México-INIFAP. Carretera Los Reyes-Texcoco km 13.5, Coatlinchán, Texcoco, Estado de México. CP. 56250. Tel. 01800 0882222, ext. 85353. (sangernan.dora@inifap.gob.mx).

${ }^{\S}$ Autora para correspondencia: djperezl@uaemex.mx.

\section{Resumen}

El presente trabajo se realizó en el ciclo primavera-verano de 2017 en Ojo de Agua, municipio de Zinacantepec, Estado de México, para evaluar la respuesta de tres niveles de gallinaza y un tratamiento de fertilización químico en cuatro cultivares de papa. Los 16 tratamientos se evaluaron en un diseño experimental de bloques completos al azar en arreglo factorial $4 \times 4$ con tres repeticiones. El tamaño de la parcela experimental se integró con tres surcos de $4 \mathrm{~m}$ de largo y 0.90 $\mathrm{m}$ de ancho. Las variables registradas fueron altura de planta, número de tallos y de tubérculos por planta, pesos de tubérculo por planta, de follaje en fresco, índice de cosecha, rendimiento por hectárea, diámetro (DT) y longitud de tubérculo (LT). Los resultados mostraron que los cv. Rosita $\left(24.38 \mathrm{t} \mathrm{ha}^{-1}\right)$ y Ágata $\left(23.85 \mathrm{t} \mathrm{ha}^{-1}\right)$, en $4 \mathrm{t} \mathrm{ha}^{-1}$ de gallinaza, produjeron los mayores rendimientos de tubérculo. Los componentes principales 1 y 2 explicaron el $69.19 \%$ de la variación total original. En 4 t ha $^{-1}$ de gallinaza hubo más tallos y mayores pesos de tubérculo, follaje en fresco y producción de tubérculo. Los cv. Rosita y Ágata sobresalieron en tallos por planta, así como en pesos de tubérculo y de follaje en fresco; la primera tuvo más tubérculos por planta. En los cv. Fianna, Ágata y Lucero se registraron los mayores índices de cosecha.

Palabras clave: Solanum tuberosum L., abonos orgánicos, análisis de componentes principales, Valles Altos del Centro de México.

Recibido: junio de 2019

Aceptado: agosto de 2019 


\section{Introducción}

La papa (Solanum tuberosum L.) es una de las principales fuentes de alimentación humana (Gopal y Khurana, 2006), después del trigo (Triticum aestivum L.), maíz (Zea mays L.) y arroz (Oriza sativa L.) (FAO, 2012). A nivel mundial se siembran más de 18 millones de hectáreas y la papa ocupa el cuarto lugar en importancia económica (FAOSTAT, 2016). En México se siembran anualmente 53107.24 ha, con una producción de 1590075.89 millones de toneladas y $32.52 \mathrm{t} \mathrm{ha}^{-1}$. Los principales estados productores son Sonora, Sinaloa, México, Veracruz, Baja California, Guanajuato, Coahuila, Nuevo León y Tamaulipas, entre otros. El Estado de México produce $175325 \mathrm{t}$ con $29.28 \mathrm{tha}^{1}$ (SIAP, 2016).

El uso y aplicación de materia orgánica ha disminuido su uso, probablemente a causa de los fertilizantes químicos que producen mayor rendimiento a menor costo. Sin embargo, en los últimos años se ha observado un mayor interés en los abonos orgánicos (Terralia, 1998) por el aporte de nutrientes a los cultivos en forma de materia orgánica (Romero et al., 2000), ya que promueve un mejor desarrollo de la planta e incrementa rendimiento y calidad del producto (Navarro, 2009). También mejora las propiedades físicas y químicas del suelo ya que el uso de fertilizantes químicos a través del tiempo ha generado pérdida de fertilidad y capacidad productiva (Trinidad, 1987).

La gallinaza es un abono orgánico concentrado y de rápida acción, contiene todos los nutrientes básicos indispensables para las plantas (Arzola et al., 1981; Yagodin et al., 1986). En varios estudios se ha observado que con gallinaza se obtuvo mayor producción y mejor calidad de tubérculo (Romero et al., 2000; Luna et al., 2016) y se incrementó el rendimiento de maíz al aumentar del fósforo disponible en el suelo (Pool et al., 2000). Las características de planta, fruto y de producción de la calabacita demostraron que el abonado con gallinaza procesada, la inoculación micorrízica o su combinación, arrojaron resultados similares a los causados por la fertilización inorgánica (Diaz et al., 2016). En Vicia faba la gallinaza y la composta de champiñón en $3 \mathrm{t} \mathrm{ha}^{-1}$ tuvieron la mejor expresión fenotípica en nudos florales, altura de planta, índice de verdor, vainas por planta, peso de vaina por planta, semillas limpias, peso de semillas limpias, peso de 100 semillas y rendimiento de grano (Orozco et al., 2016).

En la actualidad el dilema de los productores está relacionado con la ponderación entre sostenibilidad y economía; es decir, obtener una producción de calidad con mayor rendimiento. En el contexto anterior, el objetivo principal en este estudio fue evaluar la respuesta de tres niveles de gallinaza y un tratamiento fertilización inorgánico en cuatro cultivares de papa en la comunidad de Ojo de Agua, perteneciente al municipio de Zinacantepec, estado de México.

\section{Materiales y métodos}

\section{Descripción del área de estudio}

El presente estudio se desarrolló en el año 2017 en condiciones de secano en la comunidad de Ojo de agua, en el municipio de Zinacantepec, Estado de México, localizada a 99 46' 13' 'latitud norte y $19^{\circ} 12^{\prime}$ '58' longitud oeste, a $3037 \mathrm{msnm}$. En esta región predomina el clima templado subhúmedo, con una temperatura media de $28{ }^{\circ} \mathrm{C}$ en verano y $5{ }^{\circ} \mathrm{C}$ en invierno. El suelo Andosol es tipo migajón, con textura arcillo-arenoso a limoso, de color café oscuro grisáceo y con profundidad de 4 a 9 m, muy fértil (IGECEM, 2014). 


\section{Material genético}

Se utilizaron los cultivares de papa denominados Fianna, Ágata, Rosita y Lucero, proporcionadas por productores de la región Toluca-Zinacantepec (Cuadro 1).

Cuadro 1. Características agronómicas de las cuatro variedades de papa.

\begin{tabular}{|c|c|}
\hline Variedades & Descripción \\
\hline Fianna & $\begin{array}{l}\text { Planta verde de ciclo intermedio, flor blanca, tubérculo oval, cutícula } \\
\text { crema y pulpa blanca, tiene moderada resistencia a podredumbre } \\
\text { seca y a roña. }\end{array}$ \\
\hline Lucero & $\begin{array}{l}\text { Planta verde de ciclo intermedio, flor blanca, tubérculo oval, piel y } \\
\text { pulpa amarillo claro. }\end{array}$ \\
\hline Ágata & $\begin{array}{l}\text { Planta corta de ciclo intermedio, tallos extendidos, hojas grandes a } \\
\text { medianas, verdes a verdes claro, tubérculo oval, piel amarilla, } \\
\text { susceptible a Phytophthora spp. }\end{array}$ \\
\hline Rosita & $\begin{array}{l}\text { Planta corta de ciclo tardío, tubérculo oval, piel roja con ojos } \\
\text { profundos, pulpa crema, tolerante a tizón tardío. }\end{array}$ \\
\hline
\end{tabular}

Fuente: Potato Council (2011); Nivap Holland (2007).

\section{Diseño y tamaño de la parcela experimental}

Los 16 tratamientos que se formaron al combinar los niveles de cuatro cultivares y cuatro tipos de fertilización (Cuadro 2) fueron evaluados en campo en un diseño de bloques completos al azar en arreglo factorial $4 \times 4$, con tres repeticiones. La parcela experimental tuvo tres surcos de $4 \mathrm{~m}$ de largo y $0.9 \mathrm{~m}$ de ancho, se depositó un tubérculo cada $20 \mathrm{~cm}$. La unidad experimental útil fue el surco central.

Cuadro 2. Estructura de los tratamientos.

\begin{tabular}{lc}
\hline Factores de estudio & Niveles \\
\hline Cultivares & V1= Fianna \\
V2= Ágata \\
V3=Rosita \\
V4= Lucero \\
Fertilización inorgánica & $\mathrm{F} 1=154 \mathrm{~N}-356 \mathrm{P}-60 \mathrm{~K}$ \\
Fertilización orgánica con gallinaza & $\mathrm{F} 2=2 \mathrm{t} \mathrm{ha}^{-1}+\mathrm{F} 1$ \\
& $\mathrm{~F} 3=3 \mathrm{tha}^{-1}+\mathrm{F} 1$ \\
& $\mathrm{~F} 4=4 \mathrm{t} \mathrm{ha}^{-1}+\mathrm{F} 1$ \\
\hline
\end{tabular}

T1= Fianna + F1; T2= Fianna +F2; T3= Fianna + F3; , .., ; F16= Lucero + F4.

\section{Análisis fisicoquímico del suelo donde se hizo el experimento}

Los suelos que predominan en la comunidad de Ojo de Agua, en el municipio de Zinacantepec, Estado de México tienen las características que se muestran en el Cuadro 3. 
Cuadro 3. Análisis fisicoquímico del suelo.

\begin{tabular}{lccc}
\hline Características & Unidad & Valor & Clasificación \\
\hline $\mathrm{pH}$ & & 5.29 & Moderadamente ácido \\
Capacidad de intercambio catiónico & $\left(\mathrm{Cmol}^{+} \mathrm{kg}^{-1}\right)$ & 48.75 & Muy alto \\
Carbono orgánico & $(\%)$ & 3.96 & Bajo \\
Materia orgánica & $(\%)$ & 6.86 & Medio \\
Conductividad eléctrica & $\left(\mathrm{dS} \mathrm{m}^{-1}\right)$ & 0.26 & Normal \\
Nitrógeno total & $(\%)$ & 0.22 & Alto \\
Fosforo & $(\mathrm{ppm})$ & 28.69 & Medio \\
Potasio & $(\mathrm{ppm})$ & 60.25 & Bajo \\
Relación carbono/nitrógeno & $(\%)$ & 17.99 & Deficiente \\
Calcio & $(\mathrm{ppm})$ & 65.39 & Muy bajo \\
Magnesio & $(\mathrm{ppm})$ & - & Trazas \\
Sodio & $(\mathrm{pm})$ & 16.38 & Muy bajo \\
Densidad aparente & $\left.(\mathrm{g} \mathrm{cm})^{-3}\right)$ & 0.9 & Bajo \\
Clase textural & $(\%)$ arena & 69.2 & Franco arenoso \\
& $(\%)$ arcilla & 5.2 & \\
& $(\%)$ limo & 25.6 & \\
\hline
\end{tabular}

Laboratorio de Suelos de la Facultad de Ciencias Agrícolas. Datos 2017.

\section{Composición de la gallinaza}

Su análisis físico y químico se realizó en un laboratorio de la Facultad de Ciencias Agrícolas (Cuadro 4).

\section{Cuadro 4. Composición física y química de la gallinaza.}

\begin{tabular}{lcc}
\hline Característica & Unidad & Valor \\
\hline Ph & & 6.5 \\
Capacidad de intercambio catiónico & $\left(\mathrm{Cmol}^{+} \mathrm{kg}^{-1}\right)$ & 37.5 \\
Carbono orgánico & $(\%)$ & 31.2 \\
Materia orgánica & $(\%)$ & 53.8 \\
Conductividad eléctrica & $\left.(\mathrm{dS} \mathrm{m})^{-1}\right)$ & 2.83 \\
Nitrógeno total & $(\%)$ & 1.39 \\
Fosforo & $(\mathrm{ppm})$ & 207.1 \\
Potasio & $(\mathrm{ppm})$ & 860.3 \\
Relación carbono/nitrógeno & $(\%)$ & 22.5 \\
Calcio & $(\mathrm{ppm})$ & 3531 \\
Magnesio & $(\mathrm{ppm})$ & 773.4 \\
Sodio & $(\mathrm{ppm})$ & 66.4 \\
Densidad aparente & $(\mathrm{g} \mathrm{cm})$ & 0.51 \\
Clase textural & $(\%)$ arena & - \\
& $(\%)$ arcilla & - \\
\hline
\end{tabular}

Fuente: Laboratorio de Suelos de la Facultad de Ciencias Agrícolas. Datos 2017. 


\section{Desarrollo del experimento en campo}

Se realizó un barbecho y un paso de rastra el 21 de junio del 2017. Los surcos de $90 \mathrm{~cm}$ de ancho se formaron con una yunta de caballos. El 01 de junio los tubérculos se sumergieron durante cinco minutos en agua + Celeste (fludioxanil; control de Rizoctonia solani) + Bactrol (estreptomicina, control de Erwinia carotovora) y Tecto 60 (Tiabendazol, control de Fusarium spp.). La siembra manual se efectuó el 01 de Julio y consistió en depositar los tubérculos a una profundidad de 15 cm y a una distancia entre ellos de $20 \mathrm{~cm}$, después se cubrieron usando la yunta. La fertilización se realizó en dos etapas: al sembrar se aplicaron 2, 3 y 4 t ha $^{-1}$ de gallinaza y 154 N-356P-60K, en esta última se usaron $600 \mathrm{~kg} \mathrm{ha}^{-1}$ de fosfato diamónico (18 N-46 P-00 K), $100 \mathrm{~kg}$ de urea, $400 \mathrm{~kg}$ de súper fosfato de calcio simple y $100 \mathrm{~kg}$ de cloruro de potasio.

El 02 de septiembre se aplicó $200 \mathrm{~kg}$ ha de urea, $200 \mathrm{~kg}$ de cloruro de potasio y $400 \mathrm{~kg}$ de super fosfato de calcio simple. El control de maleza en postemergencia se hizo el 20 de julio con Titus CE (Rimsulfurom), aplicando en banda de 75 a $100 \mathrm{~g} \mathrm{ha}^{-1}$. Los dos aporques se realizaron el $12 \mathrm{de}$ agosto y el 2 de septiembre, para controlar maleza y prevenir plagas como gallina ciega (Phyllophaga sp.). Para evitar daños por nemátodos (Globodera rostochiensis y Meloidogyne sp.) se aplicó CYREN 5\% G (Clorpirifos etil), mezclado con fertilizantes.

Para combatir mosquita blanca (Bemisia tabaco y Trialeurodes vaporariorum), Paratrioza (Bactericera cockerelli) y gusanos trozadores (Agrotis sp., Trichoplusia ni, Spodeptera sp.), se realizaron aspersiones alternas de insecticidas. Para prevenir y controlar enfermedades como tizón tardío (Phytophthora infestans), temprano (Alternaria solani) y costra negra (Rizoctonia solani) se realizaron 12 aplicaciones con diferentes de funguicidas sistémicos y de contacto. El follaje se cortó a los 100 días de la siembra y la cosecha se hizo 20 días después de eliminar el follaje.

En 10 plantas de la parcela útil se registró altura de planta $(\mathrm{AP}$, en $\mathrm{cm}$, desde la base del tallo principal al ápice), tallos por planta, tubérculos por planta (NT), peso de tubérculo por planta (PT, en g), peso del follaje en fresco (PFF, en g), índice de cosecha (IC, cociente entre rendimiento agronómico y biológico), longitud y diámetro de tubérculo (LT y DT, en cm), y rendimiento por hectárea (Rha, en $\left.\mathrm{t} \mathrm{ha}^{-1}\right)$.

\section{Análisis estadístico}

Se realizó un análisis de varianza y cuando los valores de $\mathrm{F}$ fueron significativos se hizo una comparación de medias de tratamientos con la prueba de Tukey $(p=0.01)$. Ambas técnicas fueron descritas por Martínez (1988). El Análisis de Componentes Principales, también denominado biplot tratamiento x variable, fue descrito por Sánchez (1995); Pérez et al. (2010).

\section{Resultados y discusión}

\section{Análisis de varianza}

En el presente trabajo se observó que el área experimental fue homogénea (Cuadro 5), debido a que los efectos entre repeticiones sólo fueron significativos en peso fresco del follaje (PFF) e índice de cosecha (IC). Los efectos causados por la gallinaza (factor A) sólo se detectaron en pesos de tubérculo por planta (PT), PFF y rendimiento por hectárea (Rha), lo cual coincide con lo publicado por 
Seminario et al. (2017). Entre cultivares (factor B) en altura de planta (AP), tallos por planta (NTP), número de tubérculos (NT), PT, PFF, IC y longitud de tubérculo (LT), hubo efectos significativos $(p=0.01)$. La interacción AxB fue significativa en peso del tubérculo $(p=0.01)$ y PFF $(p=0.05)$. Estos resultados son similares a los reportados por Pérez et al. (2009); Pérez et al. (2010); Seminario et al. (2017). Los coeficientes de variación cambiaron de 6.7 (LT) a 31.48\% (Rha).

Cuadro 5. Cuadrados medios y significancia estadística de los valores de $\mathbf{F}$.

\begin{tabular}{ccccccccccc}
\hline FV & GL & AP & NTP & NT & PT & PFF & IC & Rha & DT & LT \\
\hline Repeticiones & 2 & $56.29 \mathrm{~ns}$ & $0.028 \mathrm{~ns}$ & $3.214 \mathrm{~ns}$ & $19.09 \mathrm{~ns}$ & $19595.68^{*}$ & $0.0127^{* *}$ & $1.071 \mathrm{~ns}$ & $0.227 \mathrm{~ns}$ & $1.359 \mathrm{~ns}$ \\
Tratamientos & $(15)$ & $113.43^{* * *}$ & $0.191^{* * *}$ & $60.256^{* *}$ & $41036.06^{* *}$ & $70917.93^{* * *}$ & $0.0152^{* *}$ & $36.103^{* *}$ & $0.382 \mathrm{~ns}$ & $2.284^{* *}$ \\
Gallinaza (A) & 3 & $16.31 \mathrm{~ns}$ & $0.016 \mathrm{~ns}$ & $4.753 \mathrm{~ns}$ & $88221.44^{* *}$ & $32038.85^{* *}$ & $0.0023 \mathrm{~ns}$ & $165.242^{* *}$ & $0.251 \mathrm{~ns}$ & $0.404 \mathrm{~ns}$ \\
Cultivares (B) & 3 & $452.08^{* * *}$ & $0.529^{* *}$ & $286.8^{* * *}$ & $49637.1^{* *}$ & $277566.35^{* *}$ & $0.0635^{* *}$ & $2.184 \mathrm{~ns}$ & $0.457 \mathrm{~ns}$ & $10.099^{* *}$ \\
Interacción & 9 & $32.92 \mathrm{~ns}$ & $0.137 \mathrm{~ns}$ & $3.242 \mathrm{~ns}$ & $22440.59^{* *}$ & $14994.81^{*}$ & $0.0034 \mathrm{~ns}$ & $4.364 \mathrm{~ns}$ & $0.4 \mathrm{~ns}$ & $0.306 \mathrm{~ns}$ \\
$\quad$ AxB & & & & & & & & & & \\
Error & 30 & 20.74 & 0.068 & 1.764 & 7039.4 & 4961.52 & 0.00198 & 2.476 & 0.469 & 0.523 \\
Total & 47 & & & & & & & & & \\
Media & 34.4 & 1.72 & 9.85 & 605.64 & 330.72 & 0.657 & 17.21 & 5.33 & 7.9 \\
CV (\%) & 13.34 & 15.26 & 13.48 & 13.85 & 21.29 & 6.77 & 9.1 & 12.84 & 9.15 \\
\hline
\end{tabular}

${ }^{*}=$ significativo al $0.05 ;{ }^{* *}=$ significativo al $0.01 ; \mathrm{ns}=$ no significativo; $\mathrm{FV}=$ fuente de variación; GL= grados de libertad; $\mathrm{CV}=$ coeficiente de variación; $\mathrm{AP}=$ altura de planta; $\mathrm{NTP}=$ núm. de tallos por planta; $\mathrm{NT}=$ núm. tubérculos por planta; $\mathrm{PT}=$ peso de tubérculo por planta; $\mathrm{PFF}=$ peso fresco de follaje; $\mathrm{IC}=$ índice de cosecha; $\mathrm{Rha}=$ rendimiento por ha; $\mathrm{DT}=$ diámetro de tubérculo; LT= longitud de tubérculo.

\section{Comparación de medias de gallinaza (factor A)}

El efecto de la gallinaza no fue significativo en altura de planta (AP), tallos por planta (NTP), tubérculos por planta (NT), índice de cosecha (IC), diámetro (DT) y longitud de tubérculo (LT) (Cuadro 6).

Para peso de tubérculo por planta (734.1 g, PT), peso fresco del follaje (400.71g, PFF) y rendimiento por hectárea (22.61 t ha ${ }^{-1}$, Rha) hubo mejor respuesta al aplicar $4 \mathrm{t} \mathrm{ha}^{-1}$ de gallinaza $\mathrm{y}$ esta dosis difirió estadísticamente de las menores y de la fertilización química. Ésta última y la dosis más alta difirieron en $8.38 \mathrm{t} \mathrm{ha}^{-1}$ (Cuadro 6). Carter et al. (2004) señalaron que el incremento en el rendimiento se debe a una mayor producción de tubérculos, lo cual es favorecido por un mayor contenido de materia orgánica, fósforo e intercambio catiónico disponible en la gallinaza.

\section{Cuadro 6. Comparación de medias entre abonos (factor A).}

\begin{tabular}{cccccccccc}
\hline Fertilización & AP & NTP & NT & PT & PFF & IC & Rha & DT & LT \\
\hline $\mathrm{F}_{1}$ & $35 \mathrm{a}$ & $1.7 \mathrm{a}$ & $10.17 \mathrm{a}$ & $563.21 \mathrm{~b}$ & $278.89 \mathrm{~b}$ & $0.67 \mathrm{a}$ & $14.23 \mathrm{c}$ & $5.17 \mathrm{a}$ & $7.97 \mathrm{a}$ \\
$\mathrm{F}_{2}$ & $34.84 \mathrm{a}$ & $1.68 \mathrm{a}$ & $9.08 \mathrm{a}$ & $567.86 \mathrm{~b}$ & $333.06 \mathrm{ab}$ & $0.63 \mathrm{a}$ & $15.44 \mathrm{bc}$ & $5.28 \mathrm{a}$ & $7.79 \mathrm{a}$ \\
$\mathrm{F}_{3}$ & $35.1 \mathrm{a}$ & $1.73 \mathrm{a}$ & $9.62 \mathrm{a}$ & $557.42 \mathrm{~b}$ & $310.23 \mathrm{~b}$ & $0.65 \mathrm{a}$ & $16.84 \mathrm{~b}$ & $5.52 \mathrm{a}$ & $7.71 \mathrm{a}$ \\
$\mathrm{F}_{4}$ & $32.65 \mathrm{a}$ & $1.76 \mathrm{a}$ & $10.51 \mathrm{a}$ & $734.1 \mathrm{a}$ & $400.71 \mathrm{a}$ & $0.66 \mathrm{a}$ & $22.61 \mathrm{a}$ & $5.34 \mathrm{a}$ & $8.13 \mathrm{a}$ \\
DMSH & 5.05 & 0.29 & 1.47 & 93.13 & 78.19 & 0.04 & 1.74 & 0.76 & 0.8 \\
\hline
\end{tabular}

Las medias con la misma letra dentro de cada columna son iguales estadísticamente (Tukey, $p=0.01$ ). $\mathrm{AP}=$ altura de planta. $\mathrm{NTP}=$ número de tallos por planta; $\mathrm{NT}=$ número de tubérculos por planta; $\mathrm{PT}=$ peso de tubérculo por planta; $\mathrm{PFF}=$ peso fresco de follaje; $\mathrm{IC}=$ índice de cosecha; $\mathrm{Rha}=$ rendimiento por hectárea; $\mathrm{DT}=$ diámetro de tubérculo; $\mathrm{LT}=$ longitud de tubérculo. 
Lima et al. (2000) al evaluar abonos orgánicos y fertilizantes químicos reportaron que por cada tonelada de gallinaza aplicada hubo un incremento en el rendimiento de tubérculo de $1.468 \mathrm{t}$ por encima de las $40.752 \mathrm{t}$. Zamora y Rodríguez (2008) estimaron $32 \mathrm{t} \mathrm{ha}^{-1}$ al fertilizar con abonos orgánicos y Rodríguez y Ortuño (2007) registraron de 11.53 a 15.93 t ha ${ }^{-1}$ con aplicación de micorrizas arbusculares en interacción con abonos orgánicos. Corzo y Moreno (2003) mencionaron que el principal elemento responsable de la movilización del almidón desde las hojas hacia el tubérculo es el potasio, de tal forma que una buena disponibilidad de este nutriente es decisiva para la obtención de un alto rendimiento y mejor calidad.

\section{Comparación de medias entre cultivares (Factor B)}

En Fianna se registró la mayor altura de planta $(41.89 \mathrm{~cm})$. Estos resultados son contrastantes con los de Zamora y Rodríguez (2008); Buchanan (1993) (Cuadro 7). Rosita, variedad criolla y Ágata, variedad mejorada, produjeron dos tallos por planta y difirieron estadísticamente de Fianna y Lucero. Estos resultados son similares a los de Devaux et al. (1997). Número de tallos está estrechamente relacionado con tamaño de tubérculo y con la densidad de población (Van der Zaag et al., 1990; Masarirambi et al., 2012). Rosita produjo 17.15 tubérculos por planta. Esta característica está controlada genéticamente y depende del número de tallos por planta (Estrada, 2000), en este estudio esta variedad obtuvo más tallos por planta, pero de menor longitud de tubérculo. Los resultados son contrastantes con los de Luna et al. (2015), quienes reportaron 5.91 para Rosita y 9.35 para Libertad. Pérez et al. (2009) reportaron cuantificaron de 3.75 a 5.23 en 17 genotipos de papa (Cuadro 7).

\section{Cuadro 7. Comparación de medias entre cultivares (factor B).}

\begin{tabular}{cccccccccc}
\hline Cultivares & AP $(\mathrm{cm})$ & NTP & NT & PT $(\mathrm{g})$ & PFF $(\mathrm{g})$ & IC & Rha $(\mathrm{t})$ & DT $(\mathrm{cm})$ & LT $(\mathrm{cm})$ \\
\hline Fianna & $41.89 \mathrm{a}$ & $1.61 \mathrm{~b}$ & $7.21 \mathrm{~b}$ & $598.84 \mathrm{a}$ & $305.26 \mathrm{~b}$ & $0.66 \mathrm{a}$ & $16.92 \mathrm{a}$ & $5.49 \mathrm{a}$ & $8.83 \mathrm{a}$ \\
Ágata & $36.26 \mathrm{~b}$ & $1.78 \mathrm{ab}$ & $8.05 \mathrm{~b}$ & $655.04 \mathrm{a}$ & $271 \mathrm{bc}$ & $0.71 \mathrm{a}$ & $17.88 \mathrm{a}$ & $5.49 \mathrm{a}$ & $8.5 \mathrm{a}$ \\
Rosa & $32.15 \mathrm{bc}$ & $1.98 \mathrm{a}$ & $17.15 \mathrm{a}$ & $651.49 \mathrm{ab}$ & $548.74 \mathrm{a}$ & $0.55 \mathrm{~b}$ & $17.29 \mathrm{a}$ & $5.1 \mathrm{a}$ & $6.87 \mathrm{~b}$ \\
Lucero & $27.27 \mathrm{c}$ & $1.5 \mathrm{~b}$ & $6.97 \mathrm{~b}$ & $517.21 \mathrm{~b}$ & $197.89 \mathrm{c}$ & $0.7 \mathrm{a}$ & $17.04 \mathrm{a}$ & $5.24 \mathrm{a}$ & $7.41 \mathrm{~b}$ \\
DMSH & 5.05 & 0.29 & 1.47 & 93.13 & 78.19 & 0.04 & 1.74 & 0.76 & 0.8 \\
\hline
\end{tabular}

Las medias con la misma letra dentro de cada columna son iguales estadísticamente (Tukey, $p=0.01$ ). $\mathrm{AP}=$ altura de planta. $\mathrm{NTP}=$ número de tallos por planta; $\mathrm{NT}=$ número de tubérculos por planta; $\mathrm{PT}=$ peso de tubérculo por planta; $\mathrm{PFF}=$ peso fresco de follaje; $\mathrm{IC}=$ índice de cosecha; $\mathrm{Rha}=$ rendimiento por hectárea; $\mathrm{DT}=$ diámetro de tubérculo; LT= longitud de tubérculo.

El peso de tubérculo por planta (PT) en Ágata y Rosita fue de 655.04 y 651.49 g, respectivamente y ambas difirieron estadísticamente de Lucero y Fianna. Este comportamiento podría explicarse por las diferencias genéticas que hay entre cultivares, especialmente las relacionadas con su ciclo biológico; Rosita es tardía y las otras tres son precoces. Pérez et al. (2010) reportaron valores de 627.38 a 204.13 g y Seminario et al. (2017) de 68.3 a 987.3 g (Cuadro 7). Rosita presentó el mayor peso fresco de follaje $(548.74 \mathrm{~g})$ y difirió estadísticamente de las restantes. Estos resultados contrastan con los de Perez et al. (2009), quienes registraron de 111 a $280.2 \mathrm{~g}$.

Fianna, Lucero y Ágata tuvieron índices de cosecha (66, 70 y 71\%) estadísticamente mayores a los de Rosita (50\%). Estos resultados son similares a los obtenidos por Rojas y Seminario (2014, entre 53 y 77\%). En la actualidad los fitomejoradores han dado mayor importancia al incremento del índice de cosecha ya que una mayor partición de biomasa a los tubérculos contribuirá a mayor rendimiento por hectárea. Pérez et al. (2009) reportaron valores de 38 a $72.22 \%$. 
Los resultados anteriores muestran que las papas son muy eficientes en la transformación de insumos, agua y luz a materia verde o seca y específicamente a la producción de tubérculos por planta y por hectárea. En rendimiento por hectárea los cuatro cultivares mostraron un comportamiento similar. Estos rendimientos se obtuvieron en condiciones de temporal, pero fueron menores a la media nacional $\left(24.4 \mathrm{t} \mathrm{ha}^{-1}\right)$ y estatal $\left(29.71 \mathrm{t} \mathrm{ha}^{-1}\right)$. En las sierras y Valles Altos de México el potencial de rendimiento es menor con relación a los del norte del país y en la región del Bajío (Cuadro 7).

Los cuatro cultivares fueron estadísticamente iguales en diámetro de tubérculo, resultados similares a los reportados por Luna et al. (2015). Fianna $(8.83 \mathrm{~cm})$ presentó mayor longitud de tubérculo, pero éste fue similar al de Ágata $(8.50 \mathrm{~cm})$ y ambas difirieron estadísticamente de Rosita $(6.87 \mathrm{~cm})$ y Lucero $(7.1 \mathrm{~cm})$. Esta característica es muy importante para el mercado en fresco y para la agroindustria; en la primera se requieren dimensiones entre 4.5 y $5 \mathrm{~cm}$ y para frituras deben ser mayores a $6.5 \mathrm{~cm}$. Estos valores concuerdan con los obtenidos en el presente trabajo y Ágata y Lucero se recomiendan para uso industrial, Fianna es de doble propósito y Rosita se prefiere para consumo en fresco. Con la aplicación de la escala propuesta por Macías et al. (2006), los tubérculos obtenidos en el presente estudio se clasificaron en primera categoría.

\section{Análisis de componentes principales}

Los componentes principales $1(43.21 \%)$ y 2 (25.98) explicaron el $69.19 \%$ de la variabilidad total original (Figura 1). Estos porcentajes son deseables para interpretar confiablemente las correlaciones aproximadas que se observan en el biplot, como lo sugirieron Sánchez (1995); Pérez et al. (2014), entre otros, la mayor aproximación entre cultivares y variables indica una cercana relación entre ellas.

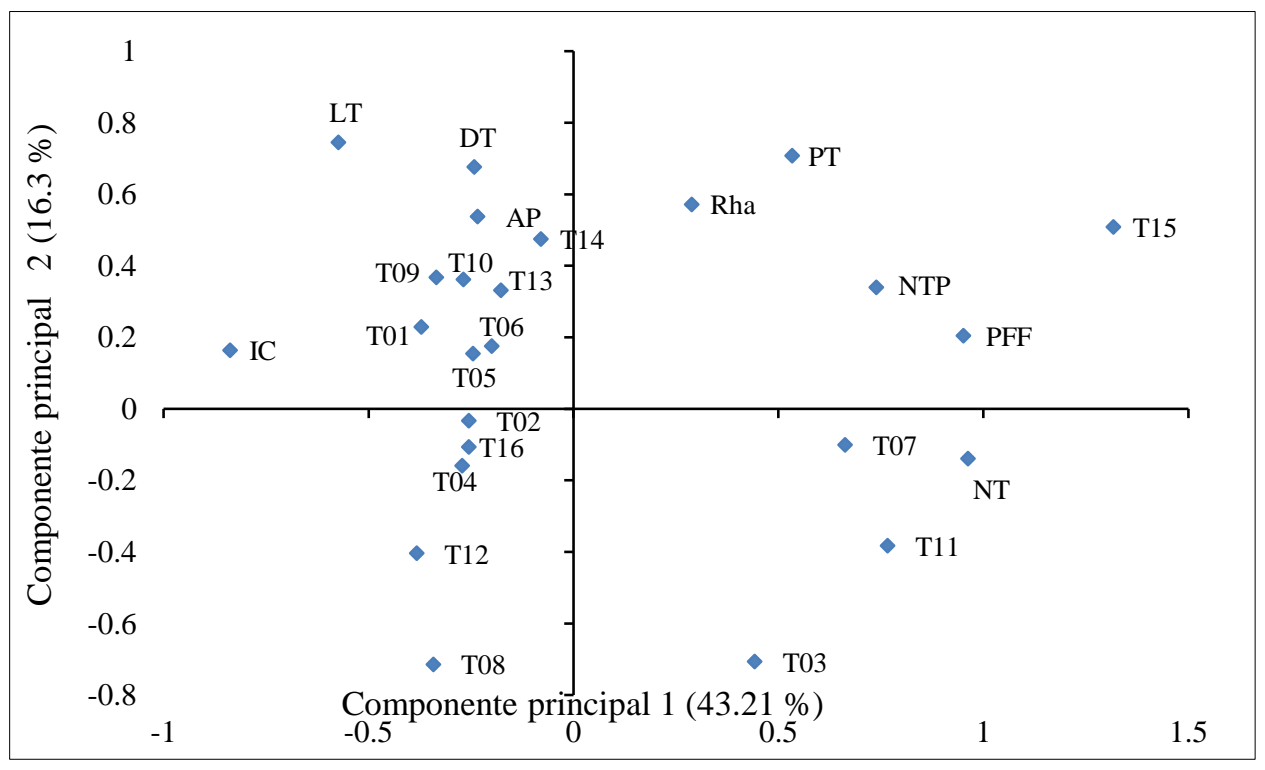

Figura 1. Biplot entre tratamientos (cultivar + fertilizante en número) y variables agronómicas (en letra) con base a los componentes 1 y 2.

El rendimiento de tubérculo se correlacionó positiva y significativamente con la mayoría de las variables. El CP1 estuvo determinada principalmente por peso de tubérculo (PT), tallos por planta (NTP), peso fresco del follaje (PFF), rendimiento de tubérculo (Rha) y tubérculos por planta (NTP). En otros estudios se han observado resultados similares (Rousselle et al., 1999; Estrada, 2000; 
Cáceres, 1971). En el Cuadrante 1 se observó que el tratamiento 15 (cv. Rosita en 4 t ha ${ }^{-1}$ de gallinaza) presentó asociación positiva con rendimiento y con sus principales componentes del rendimiento (Romero et al., 2000). Este hecho corroboró parcialmente los resultados que fueron mostrados anteriormente cuando se compararon sus promedios aritméticos con la prueba de Tukey. La gallinaza es uno de los abonos orgánicos que ayudan a mejorar las propiedades biológicas del suelo (Estrada et al., 2004; Orozco y Thienhaus, 1997), pero también contribuye al incremento en la acidez de los suelos, particularmente los de origen volcánico, como los que predominan en el área de estudio. La adaptación de las papas a Valles Altos del Centro de México ha hecho que esta especie sea una alternativa importante para los productores.

\section{Conclusiones}

Los cv. Rosita (24.38 t ha-1) y Ágata (23.85 $\left.\mathrm{t} \mathrm{ha}^{-1}\right)$, ambos en $4 \mathrm{t} \mathrm{ha} \mathrm{h}^{-1}$ de gallinaza, fueron los mejores. En $4 \mathrm{t} \mathrm{ha}^{-1}$ de gallinaza hubo más tallos y mayor peso de tubérculo, peso fresco del follaje y rendimiento de tubérculo. Los cv. Rosita y Ágata expresaron las mejores características en tallos por planta, peso de tubérculo, y peso fresco del follaje; la primera tuvo el mayor número de tubérculos por planta. Los cultivares Fianna, Ágata y Lucero sobresalieron en índice de cosecha. Las plantas más altas también produjeron tubérculos de mayor longitud y diámetro. Los componentes principales 1 y 2 explicaron el $69.19 \%$ de la variación total original.

\section{Literatura citada}

Arzola, P. N.; Fundadora, H. O. y Machado, A. J. 1981. Suelo, planta y abonado. Editorial Pueblo Educación. La Habana, Cuba. 461 p.

Buchanan, M. 1993. Study examines efficient use of compost. Cultivar-Santa Cruz. 11(1):9-10.

Cáceres, E. H. 1971. Producción de hortalizas. Herrero Hermanos, Sucesores. México, DF. $310 \mathrm{p}$.

Cadena, H. M. A. V; López, D. H.; Zabala, Q. T. E.; Flores, L. R.; Villareal, G. M.; Rivera, P. A.; Rubio, C. O.; Rocha, R. R.; Azpiroz. R. H. S.; Rodríguez, de la O. J. L.; Manzo, G. A. y Barrales, D. S. 1999. Evaluación de variedades de papa para producción de tubérculosemilla en los estados de México y Tlaxcala. Agric. Téc. Méx. 25(25):97-105.

Carter, M.; Sanderson, R. and Macleod, J. 2004. Influence of compost on the physical properties and organic matter fractions of a fine sandy loam throughout the cycle of a potato rotation. Canadian J. Soil Sci. 84(2):211-218.

Corzo, P. y Moreno, J. 2003. Manual de papa para productores. Corporación Colombiana de Investigación Agropecuaria, CI. Tibaitatá, Mosquera, Colombia. 101 p.

Devaux, A.; Vallejos, J.; Hijmans, R. y Ramos, J. 1997. Respuesta agronómica de dos variedades de papa (tuberosum y andigenum) a diferentes niveles de fertilización mineral. Rev. Latinoam. Papa. 9/10(1):123-139.

Díaz, F. A.; Alvarado, C. M.; Alejandro, A. F. y Ortiz, C. F. E. 2016. Crecimiento, nutrición y rendimiento de calabacita con fertilización biológica y mineral. Rev. Int. Contam. Ambie. 32(4):445-453.

Díaz, F. A.; Alvarado, C. M.; Alejandro, A. F. y Ortiz, C. F. E. 2017. Uso de abonos orgánicos y micorriza arbuscular en la producción de repollo. Rev. Chapingo Ser. Zonas Áridas. 16(1):15-21. 
Estrada, G.; Eugenio, M.; Peralta, C. y Ramón, J. 2004. Evaluación de dos tipos de fertilizantes orgánicos (gallinaza y estiércol vacuno) y un mineral en el crecimiento y rendimiento del cultivo de frijol común (Phaseolus vulgaris L.) variedad Dor-364, Postrera 2001. Tesis de Diploma. 1(55):11-30.

Estrada, N. 2000. La biodiversidad en el mejoramiento genético de la papa. PROINPA, CIP, CID. La Paz, Bolivia, 372 p.

FAO. 2012. Food and Agricultural Organization of the United Nations. Statistical yearbook. $366 \mathrm{p}$.

FAOSTAT. 2016. Base de datos estadísticos de la FAO. http://faostat.fao.org/site/336/ default.aspx.

IGECEM. 2014. Investigación Geográfica, Estadística y Catastral del Estado de México. Comisión de Límites del Gobierno del Estado de México. 52 p.

Lima, M. D. R.; Santos, A. T.; Espinosa, R. G. y Cerrato, R. F. 2000. Producción de papa y biomasa microbiana en suelo con abonos orgánicos y minerales. Agrociencia. 34:261-269.

Luna, M. R.; Espinosa, C. K.; Trávez, T. R.; Ulloa, M. C.; Espinoza, C. A. y Bejarano, A. A. 2015. Respuesta de variedades de papa (Solanum tuberosum, L.) a la aplicación de abonos orgánicos y fertilización química. Publicado como nota técnica en Ciencia y Tecnología. 9(1):11-16.

Macías, V. L. M.; Reyes, M. L. y Robles, E. F. J. 2006. Guía para cultivar papa en Aguascalientes. Instituto Nacional de Investigaciones Forestales, Agrícolas y Pecuarias (INIFAP)-Campo Experimental Pabellón. Aguascalientes, México. Folleto técnico núm.13. 16 p.

Martínez, G. A. 1988. diseños experimentales. métodos y elementos de Teoría. Editorial Trillas, $1^{\text {ra }}$ Ed. México, DF. 756 p.

Masarirambi, M. T.; Mandisoza, F. C.; Mashingaidze, A. B. and Bhebhe, E. 2012. Influence of plant population and seed tuber size on growth and yield components of potato (Solanum tuberosum). Inter. J. Agric. Biol. 14(4):545-549.

Navarro, G. H. 2009. Agricultura orgánica y alternativa. Editorial. Universidad Autónoma Chapingo (UACH). Texcoco, Estado de México. 271 p.

NIVAP. 2007. Instituto holandés para el fomento de ventas de patatas de siembra (por sus siglas en holandés). Catálogo de Variedades Holandesas de Papa. 286 p.

Orozco, H. L. A.; Pérez, L. D. J.; González, H. A.; Franco, M. O.; Rubí, A. M. y Aguilera, G. L. I. 2016. Efecto de Glomus fasciculatum y su relación con tres abonos orgánicos en dos cultivares de haba. Rev. Mex. Cienc. Agríc. 7(6):1423-1437.

Orozco, M. y Thienhaus, S. 1997. Efecto de la gallinaza en plantaciones de cacao (Theobroma cacao L.) en desarrollo: Agron. Mesoam. 8(1):81-92.

Pérez, L. D. J.; González, H. A.; Franco, M. O.; Díaz, H. C.; Rivera, P. A. y Ramón, G. C. 2009. Análisis de rendimiento y sus componentes en diecisiete genotipos de papa (Solanum tuberosum L.). Rev. Cienc. Agríc. Informa. 18(1):27-36.

Pérez, L. D. J.; González, H. A.; Franco, M. O.; Rivera, P. A.; Sahagun, C. J.; Balbuena, M. A.; Rubí, A. M. y Gutiérrez, R. F. 2010. Variabilidad genética, diversidad fenotípica e identificación de genotipos sobresalientes de papa. Rev. Mex. Cienc. Agríc. 1(4):579-592.

Pérez, L. D. J.; González, H. A.; Franco, M. O.; Rubí A. M.; Ramírez, D. J. R. A.; Castañeda, V. A. y Aquino, M. J. G. 2014. Aplicación de métodos multivariados para identificar cultivares sobresalientes de haba para el estado de México, México. Rev. Mex. Cienc. Agríc. 5(2):264-279. 
Pool, N. L.; Trinidad, S. A.; Etchevers, B. J. D.; Pérez, M. J. y Martínez, G. A. 2000. Mejoradores de la fertilidad del suelo en la agricultura de ladera de los Altos de Chiapas, México. Agrociencia. 34(3):251-259.

Potato Council. 2011. Manual de variedades de patatas británicas. Base de datos de variedades de patatas británicas. $255 \mathrm{p}$.

Rodríguez, K. y Ortuño, N. 2007. Evaluación de micorrizas arbusculares en interacción con abonos orgánicos como coadyuvantes del crecimiento en la producción hortícola del Valle Alto de Cochabamba, Bolivia. Rev. Boliviana. 1(5):26-51.

Rojas, M. L. P. y Seminario, C. J. F. 2014. Productividad de diez cultivares promisorios de papa chaucha (Solanum tuberosum, grupo Phureja) de la región Cajamarca. Scientia Agropecuaria. 5(4):165-175.

Romero, L. M. R.; Trinidad, S. A.; García, E. R. y Ferrera, C. R. 2000. Producción de papa y biomasa microbiana en suelo con abonos orgánicos y minerales. Agrociencia. 34(3):261-269.

Rousselle, P.; Robert, Y. y Crosnier, J. C. 1999. La patata: producción, mejorada, plagas enfermedades y utilización. Ediciones Mundi-Prensa. Madrid, España. 607 p.

Sánchez, G. J. J. 1995. El análisis biplot en clasificación. Rev. Fitotec. Mex. 18(2):188-203.

Seminario, C. J. F.; Seminario, C. A.; Domínguez, P. A. y Escalante, Z. B. 2017. Rendimiento de cosecha de diecisiete cultivares de papa (Solanum tuberosum L.) del grupo Phureja. Sci. Agrop. 8(3):181-191.

SIAP. 2016. Sistema de Información Agroalimentaria y Pesquera. Secretaría de Agricultura, Ganadería, Desarrollo Rural, Pesca y Alimentación. www.siap.gob.mx/cierre-de-laproduccion-agricola-por-cultivo.

Trinidad, S. A. 1987. El uso de abonos orgánicos en la producción agrícola. Serie Cuadernos de Edafología 10. Centro de Edafología, Colegio de Postgraduados, México. 129 p.

Van der Zaag, P.; Demagante, A. and Ewing, E. 1990. Influence of plant spacing on potato (Solaumn tuberosum L.) morphology, growth and yield under two contrasting environments. Potato Res. 33(3):313-323.

Yagodin, B. A.; Simirnov, P. and Peterburgs, K. A. 1986. Agroquímica, Tomo I y II. (Ed.). Mir Moscú. 787 p.

Zamora, F.; Tua, D. y Rodríguez, D. T. 2008. Evaluación de cinco fuentes orgánicas sobre el desarrollo vegetativo y rendimiento del cultivo de papa. Agron. Trop. 58(3):233-239. 